Ludwig M. Eichinger/Astrid Rothe (Mannheim)

\title{
Der Fall der Fälle
}

\section{Entwicklungen in der nominalen Morphologie}

\begin{abstract}
In diesem Beitrag wird an einigen Beispielen aus der nominalen Morphologie bzw. der Morphosyntax der deutschen Substantivgruppe gezeigt, wie sich in den Veränderungen in diesem Bereich, die sich über das 20. Jahrhundert hin beobachten lassen, Fragen eines langfristigen Systemwandels mit Regularitäten des Sprachgebrauchs überlagern. Im Mittelpunkt soll die Frage der Markierung der Kasus - insbesondere in den allgemein als „kritisch“ angesehenen Fällen von Genitiv und Dativ stehen. Wenn man die Daten dazu betrachtet, sieht man, dass in den meisten Fällen schon zum Anfang des 20. Jahrhunderts eine weitgehende Anpassung an die Regularitäten der Monoflexion erfolgt war, auch, dass dieser Prozess über das Jahrhundert hin fortschreitet. Bemerkenswert ist, dass insgesamt die als ,alt" angesehenen Fälle in den untersuchten Korpora geschriebener Sprache (sehr) selten auftauchen, dass aber in zunehmendem Ausmaß die daraus folgende Markiertheit in der einen oder anderen Weise funktional genutzt wird. Einen Fall eigener Art stellt in diesem Zusammenhang der Genitiv dar, der sich bei den starken Maskulina und Neutra bekanntlich dem Trend zur „Einmalmarkierung“ der Kasus an den flektierten, das Substantiv begleitenden Elementen widersetzt. Das führt zu der bekannten Orientierung dieser Formen auf die Nicht-Objekt-Verwendungen und auch zu einem auffälligen Maß an Variation in der Nutzung der entsprechenden Flexionsformen.
\end{abstract}

\section{Veränderungen in Wortschatz und Grammatik}

\subsection{Allgemeines}

Sprache verändert sich. Das allein sagt noch nicht viel. Die Frage ist ja, ob das Folgen für die deutsche Sprache und uns, ihre Sprecher und Schreiber, hat, die über das hinausgehen, dass wir uns allmählich an veränderte Regularitäten anpassen. Dabei wissen wir ohne einen genaueren Blick noch gar nicht, welchen Status eigentlich die beobachteten Veränderungen haben. Sind es Gebräuche, die in irgendeiner Weise schon vorhanden waren, jetzt aber ihren Anwendungsbereich verändert haben - oder zumindest dabei sind, ihren Anwendungsbereich zu verändern? Oder sehen wir das Ergebnis eines Wandels der Regeln und Konstruktionsweisen, die unsere Sprache grundsätzlicher prägen? Je nachdem, wie das ist, wird man sich zu diesen Veränderungen positionieren - und auch diese Positionierung wird grundsätzlicher oder weniger grundsätzlich ausfallen. Natürlich kann man sich fragen, welchen Grad an Wandel wir bei einer festgefügten, schriftlich fixierten und 
im mündlichen Gebrauch gefestigten Sprache wie dem Deutschen eigentlich erwarten und infolgedessen als „normal“ empfinden sollten. Dabei wird sich niemand wundern, dass es im Wortschatz einer Sprache Veränderungen gibt, vielmehr wird mit dieser Veränderung gerne geworben: Die gängigen Wörterbücher werben, wenn sie neu erscheinen, damit, wie viel neue Wörter in der Neuauflage gegenüber der alten aufgenommen worden seien, und dann und wann - wenn auch seltener - wird darauf hingewiesen, welche veralteten Wörter andererseits der Würde der Wörterbücher verlustig gegangen seien und nicht mehr verzeichnet würden. Die Neueinträge, die der Rechtschreibduden des Jahres 2013 verzeichnet, gehen von der Arabellion über den Leergutautomaten zum Zockerpapier. Die Streichungen betreffen Wörter wie Autocoat, Moskowitertum oder Werdarufer. Ganz offenkundig sind es im einen wie im anderen Fall Wörter, die für bestimmte aktuelle bzw. veraltete Diskurstypen stehen, formal gibt es keinen großen Hinweis auf veraltende und neue Wortbildungstypen und auch Entlehntes bzw. Bildungssprachliches gibt es hier wie dort. So steht neben der als veraltet angesehenen Suszeptibilität das neue Gentrifizieren. Wird die Sprache durch solche Verschiebungen nun reicher oder ärmer? Wenn man genau nachsieht, auf jeden Fall reicher, der Wortschatz hat zugenommen. Und auch wenn ein Teil des Aufwuchses durch Entlehnungen gedeckt wird, damit kommt die deutsche Sprache systematisch eigentlich ganz gut zurecht.

Schwerer scheinen Veränderungen der grammatischen Strukturen zu wiegen: Sie sind ja nicht so einfach abhängig von äußeren Erscheinungen, wie wir sie bei den Veränderungen im Wortschatz sehen und in vielen Fällen auch akzeptieren, ohne zu sehr darüber nachzudenken. Warum sollte sich hier überhaupt etwas ändern? Nun ist jedem, der sich mit der typologischen Stellung des Deutschen und der Entwicklung seiner grammatischen Möglichkeiten durch seine Sprachgeschichte beschäftigt hat, klar, dass sich hier mit dem Neuhochdeutschen eine grundsätzliche Schwerpunktverlagerung im engeren Kern der Grammatik, dem morphosyntaktischen System ergeben hat. Das ist wohlbekannt und gut beschrieben: Was man so generell als eine Ausweitung der grammatischen Strukturen in analytische Optionen hinein beschreibt, ist Teil einer Verschiebung, infolge derer die ehemals zentrale Stammflexion, die durch den Gebrauch selbständiger Junktoren ergänzt wurde, zu einer markierten Option wurde. Den unmarkierten Platz nehmen nun Systeme der Endungsflexion mit einem funktionalen Übergang zu grammatikalisierten Junktoren ein. Man wird auf einer abstrakten Ebene vermuten, dass es diese Prozesse sind, die im Wesentlichen zu den beobachteten Veränderungen führen. Man muss überprüfen, wo sich welche Art von Variation in diesem Bereich findet. Und man muss dann sehen, in welche Richtungen die Veränderungen weisen, die man dokumentieren kann. 


\subsection{Nominale Flexion}

Im Folgenden soll an Beispielen aus der nominalen Flexion genau diesen Fragen gefolgt werden. Anschließend kann man versuchen, etwas zu der Frage zu sagen, was hier ein Gewinn und was ein Verlust ist.

Die Flexion deutscher Substantive ist durch eine eigenständige Nutzung der Möglichkeiten der Endungsflexion gekennzeichnet. Die Eigenständigkeit gegenüber den mehr oder weniger auf Flexion gestützten Systemen der Nachbarsprachen lässt sich an drei Punkten festmachen. Der zentrale Punkt ist wohl, dass es sich um eine Wortgruppenflexion handelt, flektiert werden Substantivgruppen. Die flexivische Information ist zum zweiten über alle flektierbaren Elemente links vom Substantiv (und einschließlich des Substantivs) verteilt. Zum dritten wirkt über diesen Raum der Flexion hin in zweierlei Hinsicht das Ökonomieprinzip. Die eine Instanz dieses Prinzips wird heutzutage zumeist unter dem Begriff der Monoflexion geführt, sein anderer Teil besteht in der Nutzung eines möglichst reduzierten Inventars an Flexiven.

Die Feminina und die, was die Flexion angeht, verwandten Pluralformen kommen mit drei morphologischen Formen für die Kasus- (und Genus-) Markierung aus, den Elementen $\{-\mathrm{e}\},\{-\mathrm{er}\}$ und $\{-\mathrm{en}\}$; dazu passt, dass der unmarkierte Plural für Substantive, die im Singular als Feminina markiert sind, ebenfalls mit $\{-e n\}$ gebildet wird. Der hier praktizierte Minimalismus und die Verteilung der Informationen auch auf andere Bestandteile als das Substantiv selbst führen zu einigen Stellen, an denen das System unterdifferenziert ist. Zum einen fallen bei Feminina und bei den Pluralformen Nominativ und Akkusativ zusammen - was übrigens auch noch für die Neutra gilt -, zum anderen aber bei den Feminina auch der Genitiv und der Dativ. Da der Dativ vor allem als zweites (zumeist persönliches) indirektes Objekt und auch mit seinen freien adverbalen Verwendungen in der Satzgrammatik einen zentralen Platz einnimmt, ist das ein Problem vor allem für den Genitiv. Dessen Besonderheit zeigt sich auch in anderer Weise: Er ist zumindest bei Feminina und Plural der einzige der Kasus, der ohne Begleiter (d.h. im Singular: bei Massennomina) nur mittels einer Fügung mit der Präposition von realisiert werden kann. Das betrifft übrigens auch andere ,alte“ Genitivfunktionen, z.B. einen partitiven Objektsbezug (er trank von der Milch vs. die Milch). Dieser grundlegende partitiv-ablativische Charakter ist ja etwas, was den Genitiv insgesamt gegenüber den anderen Kasus kennzeichnet. Hier befinden wir uns dann genau am Übergang von dem endungsflexivischen zu einem analytisch junktionalen System - in gewisser Weise ist das ja ana$\log z u$ den Verhältnissen in der gesamten Substantivgruppe, die durch eine Interaktion zwischen diesen Kodierungstypen gekennzeichnet ist. Solche Übergangsfälle stehen, wenn die Wahl der Form nicht strukturell erzwungen ist - und damit Variation möglich -, dann auch stilistischer oder aussagestruktureller Variation offen (Er aß die Suppe/von der Suppe; die Hälfte der 
Suppe / von der Suppe; von der Suppe die Hälfte). Da die Präposition von, wie manche der alten einfachen (mit, zu), den Dativ regiert, könnte es scheinen, als wäre tatsächlich der Dativ dem Genitiv sein Tod.

Was wir bisher umrissen haben, ist aber nur die eine Seite der Stellung des Genitivs. Wir haben ja insgesamt von Plural und Feminina hin zu den (starken) Maskulina und den Neutra eine Zunahme der Markierungsintensität insgesamt zu bemerken, die den Genitiv ganz besonders betrifft. Die deutlich differenzierenden und phonetisch „starken“ Elemente $\{$-es $\}$ und $\{-\mathrm{em}\}$ kommen als Markierer von Genitiv und Dativ in diesen beiden Genera dazu (zudem die Nominativ-Differenzierung $\{-\mathrm{e}\},\{-\mathrm{s}\},\{-\mathrm{r}\}$ als GenusMarker). Nun ist es auffällig, dass beim Dativ zu einem Teil und beim Genitiv eigentlich durchgehend das Prinzip der Monoflexion missachtet wird: Die Genitivendung, und die des Dativ zum Teil, tritt auch am Substantiv auf - was übrigens auch für den Dativ Plural gilt, soweit das aufgrund der Form des Pluralflexivs möglich bzw. erkennbar ist. Was passiert an diesen Stellen, und wie ist eine Veränderung zu bewerten, wenn es denn eine gibt: Sind wir hier dem Sprachverfall auf der Spur?

\section{Entwicklungen im 20. Jahrhundert: Der Dativ}

\subsection{Der grundlegende Befund}

Aus den bisher gemachten einleitenden Bewertungen geht die Antwort im Prinzipiellen schon hervor. Sofern es sich um „Abrundungsmaßnahmen“ hin zu den Regularitäten der Monoflexion handelt, müsste es starke zusätzliche Gründe geben, um zu solch einer Einschätzung zu kommen. Für Verfall also eine Art der Verschlechterung des Systems - könnte man es zum Beispiel halten, wenn ,alte“ und „neue“ Formen ungeregelt nebeneinander vorkämen. Wie das ist, von welchen Zeiträumen wir sprechen und was dann daraus zu schließen sei, soll zunächst am Beispiel der Dative der starken Maskulina und der Neutra untersucht werden. Hier sind die Verhältnisse zweifellos am leichtesten zu überschauen. In allen neueren Beschreibungen, die sich dazu äußern, wird festgehalten, dass die doppelt markierten Formen mit dem $\{-e\}$-Flexiv als veraltet gelten (siehe z.B. Konopka 2012). Der letzte Satz von Schillers Räubern zeigt die Verwendung dieser Form, die an dieser Stelle zweifellos auch ihre trochäische Struktur zum Vorteil des Textes ausspielen kann.

(1) Dem Manne kann geholfen werden.

Wenn man Belege aus dem 20. Jahrhundert und vor allem aus der neueren Gegenwart ansieht, ${ }^{1}$ hat man beim Lesen entsprechender Belege den Ein-

Für diesen Beitrag wurde unter anderem ein Korpus verwendet, das für den „Bericht zur Lage der deutschen Sprache“ zusammengestellt wurde (siehe Deutsche Akademie für Sprache 
druck, dass gewisse Kontexte und dazugehörige Substantive nach wie vor ein durchaus normaler Ort für diese Form sind. Der folgende Beleg erzeugt auch durch den sonst gewählten Wortschatz (das Verfügte, zu Lebzeiten) eine textuelle Atmosphäre, in der die Form Tode als durchaus passend erscheint, auch wenn die endungslose Form an dieser Stelle auch nicht besonders auffällig wäre.

(2) Nach dem Tode der Eltern kann man sich dann ohne weiteres an das testamentarisch Verfügte halten, und alles Übel kam zu Lebzeiten ja nur daher, daß der Vater immer » zu gut «, nein, viel » zu gut « war. $\left(\right.$ Schuh 2000, S. 15) ${ }^{2}$

Wenn beide Bedingungen anders sind, sucht man aber eigentlich nach der zusätzlichen Information, die mit der merkmalhaltigen Form gegeben werden soll. Im Falle des folgenden Textausschnittes liegt die Interpretation nahe, dass es sich um ein Ironiesignal handelt.

Kaninchen verzehren letzten grünen Halm [...] Meistens liefen sie wenige Schritte vor dem Hunde fort (Braunschweiger Zeitung, 14.9.2010)

Allerdings finden sich auch durchaus alternative Verwendungen für beide Formen, bei denen man in dieser Hinsicht keinen (großen) Unterschied sieht. So benutzt die folgende dpa-Meldung - also eine eigentlich als neutral intendierte Textsorte aus dem Pressebereich - die „Langform“, während ein Zeitungsbericht, der sich offenbar auf diese Meldung bezieht, an der entsprechenden Stelle die Kurzform benutzt.

(4) Allerdings bedeute dies nur, dass sich Voyager 1 dem Rande nähert, sagte Wissenschaftler bei einem Treffen der American Geophysical Union in San Francisco. (dpa, 14.12.2010)

(5) Die amerikanische Raumsonde Voyager1 nähert sich dem Rand des Sonnensystems (Die Rheinpfalz, 15.12.2010)

und Dichtung/Union der deutschen Akademien der Wissenschaften (Hg.) 2013). Es besteht aus jeweils 10 Millionen Textwörter umfassenden Textsammlungen der Zeitscheiben 19051914, 1948-1957 und 1995-2004. Die Daten stammen aus dem Kernkorpus des Digitalen Wörterbuchs der deutschen Sprache (DWDS) an der Berlin-Brandenburgischen Akademie der Wissenschaften; für die letzte Zeitscheibe (und die folgende Zeit) wurden zudem die Korpora des Instituts für Deutsche Sprache (IDS) einbezogen. In allen Zeitscheiben sind (einigermaßen gleichmäßig) belletristische und wissenschaftliche, Zeitungs- und Gebrauchstexte (z.B. Ratgeber u.a.) vertreten. Ausführliche Auswertungen der Daten aus diesem Korpus auch in Bezug auf andere Bereiche finden sich in Eichinger (2013) und in weiteren Titeln im selben Band.

2 Belege aus dem Korpus des Akademieprojekts (vgl. Fußnote 1) sind, soweit es sich nicht um Belege aus Zeitungen handelt, mit der Angabe des Autors, des Jahres und der Seitenzahl des entsprechenden Textes versehen. Die Belegstellenangaben, die den Korpusbelegen beigefügt sind, entsprechen den Angaben, die das zugrunde gelegte Korpus liefert. Wenn die Angaben dabei nicht den Seitenzahlen der gedruckten Werke entsprechen, liegt das daran, dass das Korpus teilweise auf elektronischen Fassungen der Texte mit anderer Zählung basiert. 


\subsection{Differenzierung nach Lexemtypen}

Wenn man daraufhin die Befunde aus den Korpora zu den Jahren nach 1900, um 1950 und um 2000 betrachtet, ergibt sich eine klare Entwicklung und ein in mancherlei Hinsicht zu differenzierendes Bild. Der klare - und auch so zu erwartende - Gesamtbefund lautet: die Verwendung der $\{\mathrm{e}\}$-haltigen Form nimmt über das Jahrhundert hin dramatisch ab, in einer ganzen Reihe von Fällen finden sich keine oder nur noch ganz vereinzelte Belege für diese Form. Das gilt gleichermaßen für Substantive wie Bad oder Mord, bei denen diese Verwendung zu Beginn des letzten Jahrhunderts noch sehr häufig war und etwa 85 Prozent bzw. 90 Prozent der entsprechenden Kasusformen ausmachte, wie für welche wie Kopf, bei denen die Form Kopfe auch schon damals nur etwa ein Drittel der für dieses Wort belegten Dativ-Belege darstellt. Schon wenn man nur diese drei Fälle nebeneinanderstellt, sieht man, dass sie unterschiedliche Entwicklungsgeschichten haben.

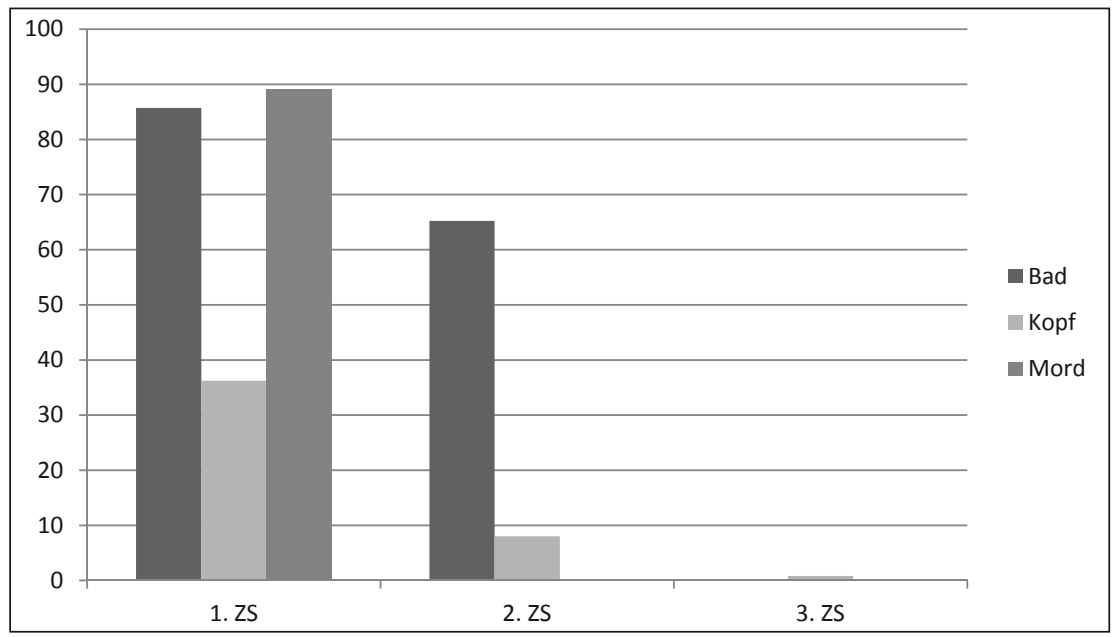

Abb. 1: Entwicklung der Dativ-Flexion bei Bad, Kopf, Mord (prozentualer Anteil der Treffer mit Dativ-e, Suchanfrage: dem/einem + Nomen)

Bei einem Wort wie Bad, bei dem sich die Langform auch bis in die 1950er hinein gehalten hat, kann man davon ausgehen, dass das Alltäglichwerden des Bades im Haus und der Frei- und Schwimmbäder zu jener „Normalität" der systematisch zu erwartenden endungslosen Form geführt hat, die dem Alltag unserer Sprache entspricht.

(6) Ich schütte das Kind mit dem Bad aus, weil das Kind vom Bad dreckig wurde. (Kraus 1914, S. 14-17 bzw. 258050)

(7) 8. Sollen zu einem Bade Zusätze gemacht werden (Absatz 3), so muß dies genau nach Vorschrift des Arztes und erst dann geschehen, wenn das Bad fertig ist. (Körting 1907, S. 258050) 
(8) Man darf das Kind nicht mit dem Bade ausschütten, indem man jene an sich richtige Erkenntnis übertreibt.

(Schumpeter 1912, S. 258050)

(9) Wir haben das Gefühl, in einem Bad aus heißem Lehm zu liegen, so schwer, daß wir unfähig sind, uns zu rühren.

(Riemkasten 1953, S. 258050)

(10) Es ist wichtig, das Kind vor dem Bade einmal vom Kopf bis zum Fuß zu besichtigen, um Hautausschläge oder sonstige Unregelmäßigkeiten festzustellen. (Bundesverband der deutschen Standesbeamten e.V. (Hg.) 1956, S. 258050)

(11) Ihr aber wollt das Kind mit dem Bade ausschütten, und statt der individuellen Freilassung, die immer wieder geübt wird, laßt Ihr Alles frei, auch wenn es gern sorgenlos unfrei bliebe

(Niebelschütz 1949, S. 258050)

(12) Bo kam aus dem Bad, wo er sich kaltes Wasser über den Kopf hatte laufen lassen. (Jentzsch 1996, S. 157)

Dass diese Entwicklung schon um 1900 systematisch weit vorangekommen war, zeigt der niedrige Ausgangswert für die Form Kopfe, von dem aus es kontinuierlich nach unten geht. In der dritten Zeitscheibe ist die Verwendung der Langform stilistisch als überneutral markiert.

(13) Er stieß stets mit dem Kopf an die niedrige Decke.

(Tucholsky 1914, S. 43 bzw. 258050)

(14) Kein Herr rauchte, keiner behielt den Hut auf dem Kopfe, selbst nicht im zugigen Lift, wenn eine Dame zugegen war.

(Lehmann 1913, S. 42986)

(15) Da wurde ihr Gesicht zornig, blähte sich, daß alle Falten verschwanden, die Augen traten aus dem Kopf, aus ihren runden Klagehänden wuchsen Krallen, die auf die Kapsel losfuhren, der Mund öffnete sich zu einem Schrei, blieb offenstehen, sie selbst verharrte wie vom Schlag gerührt in dieser Haltung, bis eine Tür aufging, ein Herr, dem die Zigarette erbärmlich schief im Munde hing, zu ihr hintrat und sagte (Walser 1957, S. 258050)

(16) Und der Pächter schaute, daß ihm die Billardkugelaugen fast aus dem Kopfe sprangen. (Matthiessen 1949, S. 258050)

(17) So saßen sie, bis es klingelte und Doktor Massini hereinkam, mit einer Pelzmütze auf dem Kopf und Schnee hinter sich herschleppend. (Widmer 2004, S. 124)

(18) Dieser alte, gestiefelte, gerockte, mit einer Krawatte angethane Herr mit den rund abgeschnittenen Haaren und dem Kastorhut auf dem Kopfe - war die Frau Gräfin Itzenplitz wie sie leibte und lebte, und selbst die Reitgerte in der Hand fehlte nicht, obgleich von Reiten nicht die Rede war. (de Bruyn 1999, S. 92) 
Die Form Morde stellt irgendwie ein Gegenmuster zu Bad dar: 1900 noch fast ausnahmslos die Dativ-Form von Mord, ist sie schon 1950 völlig verschwunden.

(19) Was veranlaßte Sie, der Angeklagten das Ehrenwort abzunehmen, daß sie an dem Morde ihres Mannes nicht beteiligt sei?

(Friedländer 1913, S. 258050)

(20) Hat Ihnen Ihre Schwester gesagt, ob und inwieweit sie an dem Mord beteiligt war, ganz besonders ob sie den Mord mit Rieß vorher verabredet hatte? (Friedländer 1913, S. 258050)

(21) aber es hat keinen Sinn mehr, Gastmann zu belästigen, da doch hinter dem Mord Mächte stehen, die weder mit unseren braven Schweizer Industriellen noch mit Gastmann etwas zu tun haben.

(Dürrenmatt 1952, S. 258050)

(22) Ich habe seit dem Mord nichts in unserem Haus verändert.

(Mittelstaedt/Beilke 2002, S. 6 bzw. 1468)

Was sind nun typische Fälle für heutzutage noch in nennenswertem Ausmaß erhaltene Fälle der $\{\mathrm{e}\}$-haltigen Dativ-Flexion? In der von uns untersuchten Beispielliste gibt es bei den Substantiven Jahr, Mund und Tod Belege dieses Typs in etwa 20 Prozent der einschlägigen Fälle. Außer dem Flexionstyp scheinen diese Substantive nicht viel gemein zu haben.

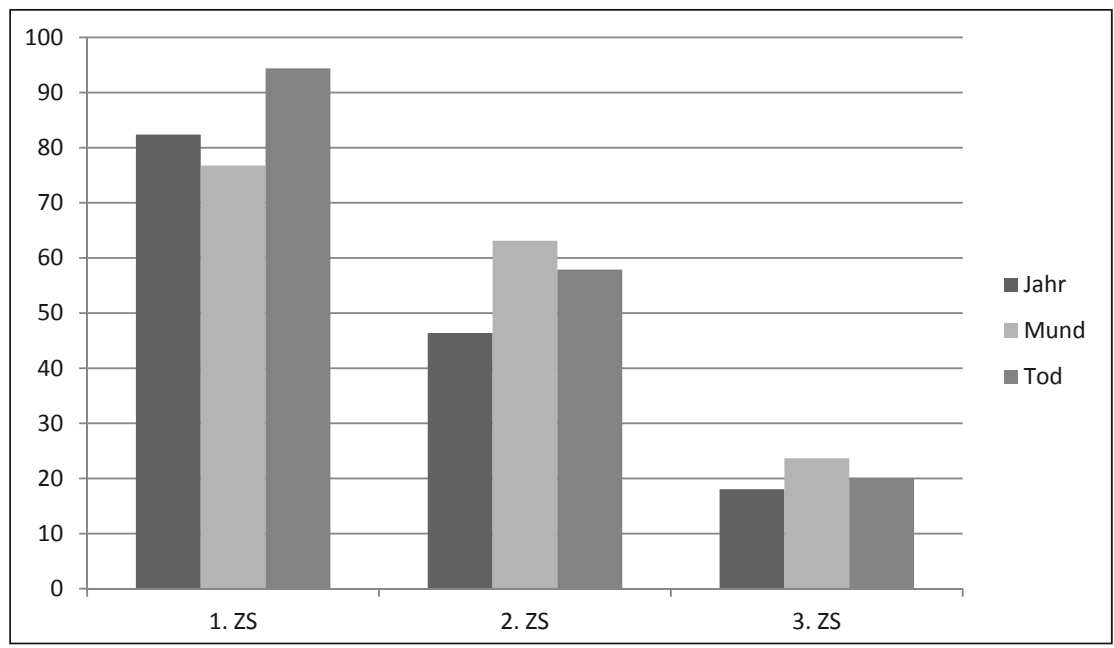

Abb. 2: Entwicklung der Dativ-Flexion bei Tod, Jahr, Mund (prozentualer Anteil der Treffer mit Dativ-e, Suchanfrage: dem/einem + Nomen)

Auffällig ist zudem, dass die Langform im Vergleich mit den anderen Substantiven bei diesen Fällen an relativer Bedeutung gewinnt, obwohl ihr Anteil insgesamt natürlich auch sinkt. Woran liegt das? Man kann diese Verteilung als ein Beispiel dafür ansehen, dass die „alten“ Formen nicht einfach 
undifferenziert verschwinden, sondern als markierte Formen für bestimmte Zwecke funktionalisiert werden. So ist bei Tode sicherlich diese Form eine Möglichkeit, stilistische Überneutralität („Feierlichkeit") in einschlägigen Kontexten zu verdeutlichen.

(23) 2. Akt. Dem Oberpriester Nourabad, der Leila die Heiligkeit ihres Eides vor Augen führt, vertraut sie an, dass sie als Kind einmal unter Lebensgefahr einen Fremden vor dem Tod gerettet habe und noch die Schmuckkette besitze, die sie von ihm als Dank erhalten hat. (Fath 1998, S. 1927)

(24) Wo er doch im engsten Kreise warnte: » Lieber Millionen Menschen vor dem Tode retten, als wie einen Banditen leben lassen, der also uns dann die Toten bringt. (Brussig 1995, S. 254)

Etwas Ähnliches - die Entwicklung verläuft ja auch ganz parallel - lässt sich auch für die Form Jabre sagen. Die ,,alte“ Form - die Variante mit Dativ-e nach definitem Artikel - wird in der dritten Zeitscheibe nur in bestimmten Kontexten verwendet, sie kommt ausschließlich zusammen mit einer Jahreszahl und in beinahe allen Fällen nach der Präposition aus vor:

(25) Jürgen Böttchers Dokumentarfilm Wäscherinnen aus dem Jahre 1972 gab einen guten Einblick in diese weibliche Arbeitswelt und ihren durchaus männlichen Komment. (Engler 1999, S. 223)

(26) Nach einem Beschluss der KMK aus dem Jahre 1988 können Fachgymnasien für folgende Gebiete eingerichtet werden [...].

(Schaub/Zenke 1995, S. 801)

(27) Seit dem Jahr 2000 schreibt das Unternehmen, dessen Hauptgesellschafter der Tierschutzverein ist, schwarze Zahlen.

(Süddeutsche Zeitung, 16.4.2002)

(28) Aus dem Jahr verfüge ich auf Grund meines Langzeitgedächtnisses noch über eine größere Zahl von einzelnen Erinnerungen.

(DIE WELT, 7.3.2002)

(29) Sawallisch wird in dem Jahr 80 Jahre alt. (DIE WELT, 10.1.2001)

(30) Macht und Überleben « aus dem Jahr 1962 entfaltet hat.

(Schuh 2000, S. 253)

Ähnliches gilt für die zweite Zeitscheibe, allerdings ist im Vergleich zur dritten Zeitscheibe die Variation der vorangehenden Präposition vor der Langform noch größer:

(31) Wir wissen, so erstaunlich dies klingen mag, erst seit dem Jahre 1875 um dieses tiefste Lebensgeheimnis Bescheid.

(Buddenbrock 1953, S. 258050)

(32) Auch in der Festsetzung der Epoche für diese neue Unsicherheit können wir uns auf Rothfels berufen und anerkennen, daß etwa mit dem Jahre 1917, mit dem Kriegseintritt Amerikas, und mit der russischen 
Revolution die geschichtliche Konstellation wirklich universal und der Völker- und Staatenkonflikt durch gesellschaftliche Gegensätze durchzogen und durchkreuzt wurde. (Heimpel 1953, S. 258050)

(33) Die Arbeitsproduktivität soll im Jahre 1953 in der Industrie um 10,2 Prozent, im Eisenbahnverkehr um 5,5 Prozent, im Bauwesen um 6 Prozent gegenüber dem Jahre 1952 gesteigert werden. (Archiv der Gegenwart 23 (1953), S. 3874 bzw. 258050)

(34) Im 1. Schuljahr, dem Jahr der Eingewöhnung, fällt dem Turnunterricht zum großen Teil die Aufgabe zu, die Kinder auf dem Wege vom Einzelspiel zum Gemeinschaftsspiel weiterzuführen.

(Borrmann/Mügge 1957, S. 258050)

(35) Der Zeitfahrer kehrt nach mancherlei Gefahren aus dem Jahr 802701 zu seinen gegenwärtigen Freunden zurück, eine Blume in der Hand, die auf der ganzen jetzigen Erde nicht vorkommt.

(Bloch 1954, S. 258050)

(36) Seit dem Jahr 1946 sei die Industrieproduktion um 40\% gestiegen. (Archiv der Gegenwart 21 (1951), S. 2767 bzw. 258050)

Etwas anders ist das bei der Verwendung der Form Munde. Für die Erklärung des Verlaufs bei diesem Wort ist es hilfreich, sich anzusehen, inwieweit hier Bindungen in Kollokationen eine Rolle spielen. Wenn man für eine Reihe ,einschlägiger" Substantive den relativen Anteil der in phraseologischen Wendungen vorkommenden an allen $\{\mathrm{e}\}$-haltigen Formen vergleicht, sieht man eine ganz erhebliche Steigerung dieses Anteils über das 20. Jahrhundert und auf jeden Fall seine zweite Hälfte hin.

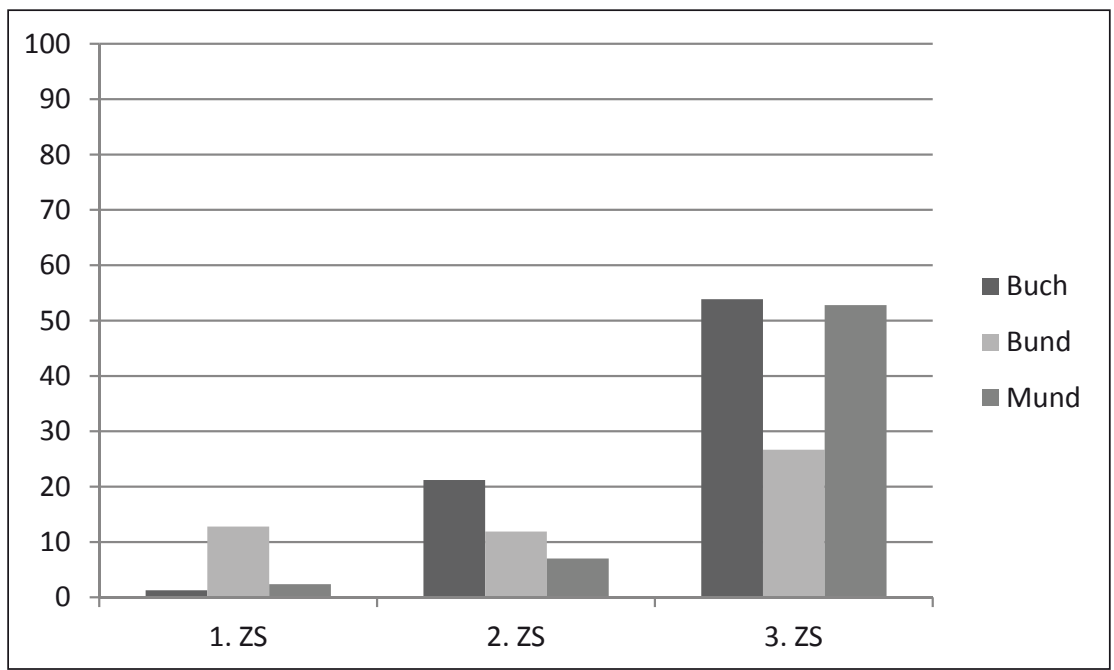

Abb. 3: Anteil an Dativ- $e$ in Phraseologismen gegenüber Dativ- $e$ nach dem/im (in Prozent) 
Gerade bei dem Wort Mund ist diese Entwicklung sehr deutlich. In ihrer Mehrheit sind es die in den folgenden beiden Belegen vorkommenden festen (präpositionalen) Wendungen, von denen dieses Bild geprägt wird.

(37) Und diesen Feinden hat der kleine Riese nach dem Mund geredet. (Walser 1998, S. 191)

(38) Ein solches Blabla aus dem Munde von Rechtskundigen kann nur Vernebelungsfunktion haben. (Hannover 1998, S. 193)

Bei Buch geht der Zuwachs auf das Konto einer Art verbonominaler Fügungen mit den Verben schlagen und stehen:

(39) Noch stärker schlagen jedoch die Verluste durch mangelhaftes Website-Design zu Buche. (DIE WELT, 10.1.2001)

(40) [...] und als bestes Ergebnis bei Grand-Slam-Turnieren stand bisher das Erreichen des Achtelfinales bei den Internationalen Französischen Meisterschaften im vergangenen Jahr zu Buche.

(Frankfurter Allgemeine Zeitung, 23.1.1995)

Diese festen Verwendungen führen auch zu adverbial-vergleichenden Phraseologismen:

(41) Ein Sommertag wie er im Buche steht, denkt Koberling, dröhnt die Treppe hinunter und reißt die Küchentür auf.

(Hermann 1998, S. 187)

Aber auch bei Wörtern wie z.B. Rand machen Fügungen vom Typ zu Rande (kommen) in den IDS-Korpora des Jahres 2010 weit über 90 Prozent der Belege aus. Und wenn man heutzutage die Dativ-Form (dem) Bade liest, kann man mit hoher Wahrscheinlichkeit davon ausgehen, dass ein Kind mit demselben ausgeschüttet wird.

(42) Agrar-Reform mit Augenmaß [...] Allerdings gilt es, nicht gleich das Kind mit dem Bade auszuschütten.

(Nürnberger Nachrichten, 19.11.2010)

In diese Kategorie gehören auch adverbiale Fügungen vom Typ von/ zu/ nach Hause:

(43) Oder wenn ich nach Hause komme und noch nicht schlafen mag. (Suter 2004, S. 75)

Das führt dann auch zu Idiomatisierungen wie:

(44) Und in einem Kellerraum haust der sächsische Rudloff, von Hause aus Senker, der beim Holundbringdienst arbeitet und seinerzeit mit zwei Oberschenkelknochen für die deutsche Einheit demonstrierte, um seine Verhaftung zu provozieren. (Braun 2003, S. 44) 
Das passt dazu, dass in bestimmten Fällen das flexivische Muster zur Differenzierung in Fällen von Polysemie oder Homophonie dient, so bezieht man sich mit der Nutzung der merkmalhaften Form von Haus zumeist auf die Bedeutung, Herkunft, Familie, Firma, Institution` o.Ä.

(45) Ein Panzer ist so ziemlich das letzte, was man mit Philipp Lahm, 20, assoziiert, es sei denn, er käme aus dem Hause Lego oder Playmobil. (Süddeutsche Zeitung, 20.2.2004)

(46) In einem langen und blutigen Krieg erkämpften sie unter der Führung von Moritz von Nassau aus dem Hause Oranien gegen die Spanier ihre Freiheit. (Schwanitz 1999, S. 120)

Man kann an diesen Beispielen sehen, wie man die generelle Entwicklungsrichtung und die Unterschiede der Verläufe bei den einschlägigen Substantiven interpretieren kann und wie sie in die Systementwicklung eingebettet ist. In gewisser Weise prototypisch erscheint die Entwicklung einer Reihe weniger stilistisch hervorgehobener Substantive. Hier sieht man, dass über das Jahrhundert hin gewechselt hat, was als die normale Flexionsform zu gelten hat. Bei diesen Substantiven treten auch die bisher dokumentierten Umfunktionalisierungen nur marginal auf (vgl. Abb. 4).

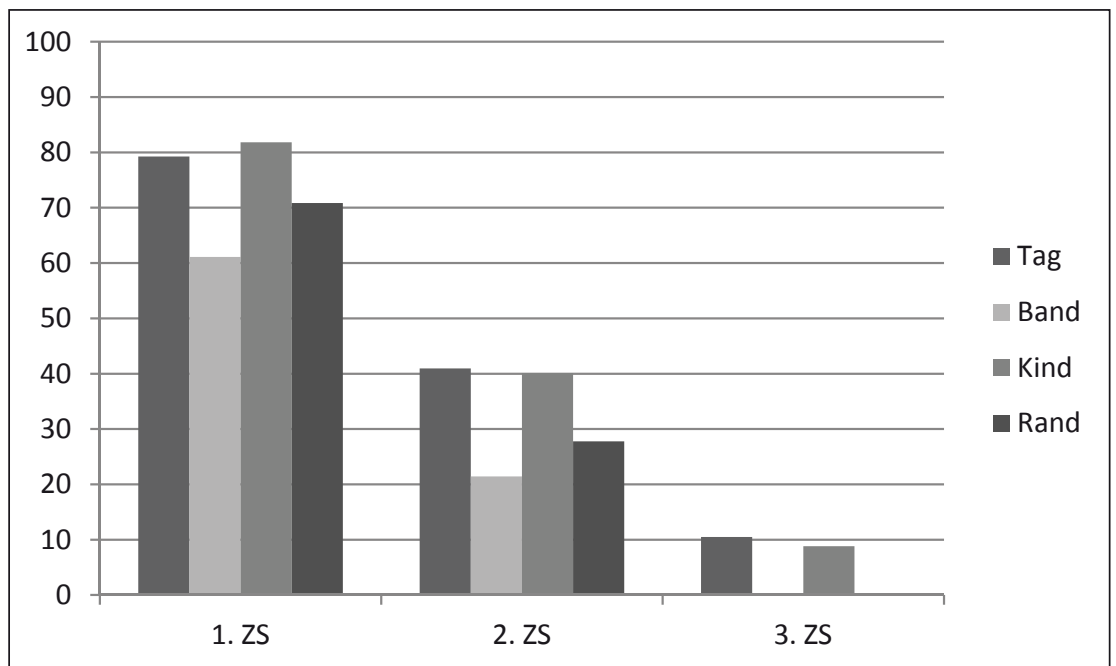

Abb. 4: Entwicklung der Dativ-Flexion bei Tag, Band, Kind, Rand (prozentualer Anteil der Treffer mit Dativ-e, Suchanfrage: dem/einem + Nomen)

Bis hierher lassen sich drei Punkte festhalten. Zum einen ist der Sog hin zur systemgerechten Markierung des Dativs durch flexivische Mittel, die bei Determinatoren und Adjektiven auftreten, über das 20. Jahrhundert hin wirksam. Von ihm werden im Prinzip alle einschlägigen Lexeme weitgehend erfasst - allerdings doch in unterschiedlichem Ausmaß. Zum zwei- 
ten nutzen einerseits Lexeme, die einen überneutralen Charakter behalten haben, soweit sie in entsprechenden Kontexten auftreten, die merkmalhafte Form zur Stärkung dieser stilistischen Markierung. Andererseits setzen Lexeme, die einem stilistisch unmarkierten Normalfeld angehören, die merkmalhafte Form in festen und phraseologischen Wendungen ein und nutzen sie so zur Markierung dieses Tatbestands. Zum dritten werden die merkmalhaften Formen zur Basis von Prozessen der Lexikalisierung und Grammatikalisierung.

\subsection{Differenzierung nach Texttypen}

Wenn man die beobachteten Funktionen betrachtet, die von den markierten $\{\mathrm{e}\}$-haltigen Formen übernommen werden, erscheint es wahrscheinlich, dass unterschiedliche Texttypen die Veränderungen und Verwendungstypen in unterschiedlichem Ausmaße zeigen. Am unmittelbar einleuchtendsten erscheint das vielleicht bei den stilistisch besonders markierten Fällen, die logischerweise spezifischere Verwendungsbedingungen zeigen sollten. So sieht die Sache z.B. für das Wort Tod folgendermaßen aus (vgl. Abb. 5):

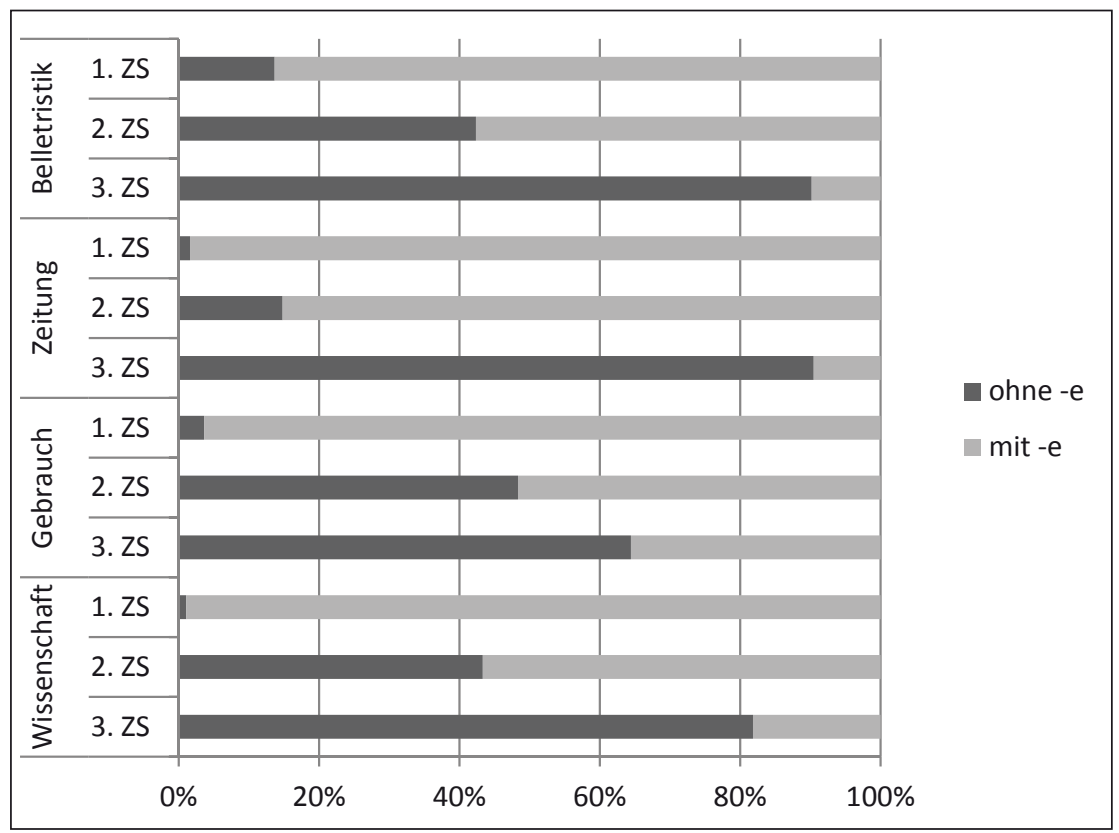

Abb. 5: Dativ von Tod, Verteilung nach Textsorten

Natürlich gilt auch bei anderen Wörtern die generelle Entwicklungsrichtung, allerdings stellt sich die genaue Verteilung bei einem Substantiv wie Jahr doch anders dar (vgl. Abb. 6). 


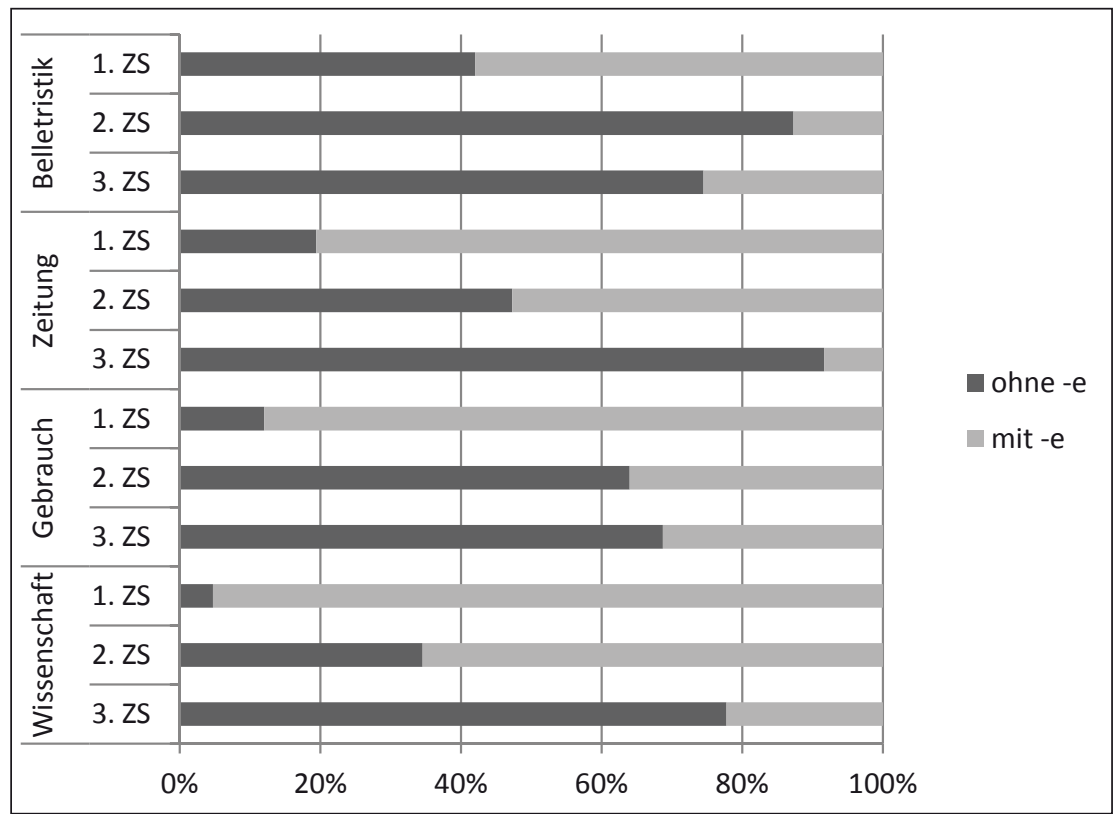

Abb. 6: Dativ von Jabr, Verteilung nach Textsorten

Wo der Systemsog mit der Neigung zur stilistischen Gehobenheit zusammenkommt, wie bei Tod, ist das Bild noch klarer als in den anderen Fällen. Zu Anfang des 20. Jahrhunderts stellt die Langform den Normalfall dar, die endungslose Form kommt nur ganz marginal vor. Das hat sich bis zum Ende des Jahrhunderts umgekehrt, wobei die $\{\mathrm{e}\}$-haltige Form in fachlichen und alltäglichen Alltagstexten im Rahmen einer Art „Distanzstilistik“ einen gewissen Platz behält. In den Zeitungen spielt dieser Faktor offenbar kaum eine Rolle, und bei literarischen Texten gibt es offenbar die freie Wahl, die stilistisch markierte oder unmarkierte Form zu nutzen.

\section{Entwicklungen im 20. Jahrhundert: Der Genitiv und die Monoflexion}

Ganz offenkundig übertreibt der Genitiv der starken Maskulina und der Neutra im Hinblick auf die Markierung des Genitivs: Systematisch tritt hier das Flexiv neben dem Artikel bzw. Adjektiv auch am Substantiv auf. ${ }^{3}$ Da das ein sehr starkes Signal in einer sehr deutlichen phonetischen Form ist, gibt es manchmal Fälle, wo das zu viel des Guten zu sein scheint. An einer Stelle

Zur Unter- und Überdifferenzierung im Bereich des Genitivs siehe u.a. Eichinger (2012) und weitere Titel im selben Band. 
ist die so entstehende Übermarkierung in einer Weise reduziert, die so gar nicht zu den sonstigen Kodierungsgewohnheiten der Monoflexion passt. Es wird am Adjektiv reduziert, und nicht an der Endung des Substantivs, wie wir das beim Dativ gesehen haben. Diese Veränderung hat einen längeren Weg hinter sich. In Goethes Werther zum Beispiel findet sich der folgende Beleg:

(47) [... ich merke, das Schicksal hat mir harte Prüfungen zugedacht. doch gutes Muts! ein leichter Sinn trägt alles

(Goethe, Werke; Werther, (Erstv. 1787), 1982, Bd. 6, S. 60)

Erkennbar ist das aber schon ein Übergangsstadium, in dem sich in einigermaßen festen Wendungen eine alte Form hält. Dafür spricht auch, dass sich Belege genau dieses Typs in den Grimm'schen Märchen und in Sagen finden, Texten also, denen Alter und Gesprochenheit als Charakteristika eingeschrieben sind.

(48) Doch sei gutes Mutes, ich will mich deiner annehmen und dir sagen, wie du zu dem goldenen Pferd gelangst.

(Hausmärchen; Der goldene Vogel, (Erstv. 1819), 1978, S. 324)

Selbst eher alltägliche Grammatiken und Sprachlehren der Zeit kommentieren das Problem dieser Übermarkierung und plädieren für die morphologische Entlastung:

Die Biegungssylben sind von dem Artikel, der, die, das, entlehnt; daher man, wie es auch einige thun, im Genitiv eigentlich sagen sollte: gutes Weins. Weil aber der Genitiv an dem Substantiv durch das s deutlich genug bezeichnet ist, so ist es nicht nöthig, das Kasuszeichen dem Adjektiv zu geben, sondern man zeige durch die Sylbe en nur an, dass das Adjektiv dem Substantiv einverleibt wird. (Klügel 1809, S. 91$)^{4}$

Man sieht auch, dass eine Reihe der Fügungen mit der doppelten Genitivmarkierung durch intertextuelle - vor allem biblische - Bezüge gestützt werden. Die damit verbundene stilistische Markierung wird auch in dem einzigen Beleg genutzt, der sich im Korpus findet - es wird an dieser Stelle die Bibel zitiert. Im weiter fortlaufenden Text tritt dann in einer inhaltlichen Paraphrase derselben Stelle die erwartbare Form mit der schwachen Adjektivendung ein.

Zu dieser Frage und zur historischen grammatischen Diskussion der Flexion attributiver Adjektive insgesamt siehe Takada (1998, S. 181 ff., insbesondere S. 188-189); vgl. z.B. auch die Feststellung in Heinrich Brauns (1775) Grammatik, mit der die Geltung der Gottsched'schen Normen für Bayern festgeschrieben wird: „Bey den Beywörtern [...] darf man das s im Genitiv in kein n verwandeln. Z.B. Ein Glas voll guten Weins; ist falsch, es soll heißen: voll gutes Weins.“ (S. 135).; vgl. auch das Zitat aus der Heyse-Grammatik von 1838 in Eichinger (2011, S. 53). 
(49) Ich weiß wohl, daß geschrieben steht: ,Ich habe es den Weisen verborgen; den Ungelehrten, den Kindern und Unmündigen, denen, die arm an Geist und reines Herzens sind, dagegen zu wissen getan!' [...] Aber wenn man bedenkt, wie Jesus die Armen an Geist und die Einfältigen, wenn sie nur reinen Herzens waren, besonders liebte, so wird man nicht ohne Nachsicht sein. (Hauptmann 1910, S. 258050)

Vielleicht überraschender als die Wahl der schwachen Option bei den Adjektiven, bei denen sie ja immerhin im Prinzip vorgesehen ist, sind die entsprechenden Verwendungen im Bereich der artikelähnlichen Wörter. Ein klassisches Beispiel ist die im Laufe des 20. Jahrhunderts aufgekommene Form diesen Jahres neben (nach wie vor weithin dominantem) dieses Jahres. ${ }^{5}$

\begin{tabular}{|l|c|c|c|}
\cline { 2 - 4 } \multicolumn{1}{c|}{} & 1. Zeitscheibe & 2. Zeitscheibe & 3. Zeitscheibe \\
\hline diesen Jabres & - & - & 13 \\
\hline dieses Jahres & 99 & 139 & 157 \\
\hline
\end{tabular}

Es ist ganz klar, dass diese Erscheinung nur bei bestimmten Substantivtypen auftritt, und wenn man die Belege im Einzelnen durchsieht, kann man feststellen, dass auch diese Kombinationen in einem relativ eng begrenzten Raum von Konstruktionen vorkommen. ${ }^{6}$ Typische Kollokationen sind Verbindungen mit den geradezu präpositionsähnlichen Substantiven Anfang, Mitte oder Ende, wie in dem folgenden Beleg:

(50) Anfang diesen Jahres erlitt ein 49-Jähriger einen Leberschaden (Süddeutsche Zeitung, 16.4.2002)

Nicht überraschend ist, dass sich diese Verwendung dann auch auf die deiktische Festmachung von Tagen, Monaten und Jahreszeiten auf das jeweilige Jahr erstreckt.

„Wo waren Sie am fünfundzwanzigsten März diesen Jahres?" fragte Willi. (Jentzsch 1996, S. 132)

Wenn sich diese Verwendung auf andere semantische Gruppen bezieht und das ist nur in den letzten beiden Zeitscheiben belegt - dann handelt es

5 Eine ähnliche Verteilung findet sich laut einer Untersuchung von Sahel (2005, S. 490) in 621 Belegen des Jahrgangs 2005 der Berliner Zeitung: Die Form dieses Jahres macht 90 Prozent der Belege aus, die Form diesen Jahres 10 Prozent (vgl. Sahel 2005, S. 489). Auch die Auswertung eines Korpus von Ausgaben der Zeit stützt diese Tendenz: Von 1946 bis 2009 nimmt die Form diesen Jabres kontinuierlich zu.

6 Dass diesen Jahres hier in Analogie zu folgenden Jabres als eine Art demonstrativen (Pronominal-) Adjektivs (Formulierung nach Wiese 2009, S. 184) verwendet wird, leuchtet ein und wird auch von Strecker (2006) dargelegt (siehe dazu auch Stenschke 2007 und Heringer 2012). Die Parallelformulierung diesen und des folgenden Jahres findet sich in den Korpora nicht. 
sich um eine Spezifikation einer Modifikation der „Art und Weise“, wie sie in genereller Weise durch das Element \{solch\} ausgedrückt wird. So sind für die fünfziger Jahre die folgenden Verbindungen belegt:

(52) Die Elastizität der Betriebe ist groß genug, um Nachfrageänderungen diesen Umfangs entsprechen zu können.

(Archiv der Gegenwart 24 (1954), S. 4726)

(53) eine Maßnahme diesen Charakters zu treffen

(Archiv der Gegenwart 24 (1954), S. 4627)

Entsprechende Belege aus der dritten Zeitscheibe sind folgende:

(54) eine Kollision diesen Kalibers dürfte auch in der Milliarden Jahre langen Geschichte des Sonnensystems Seltenheitswert haben.

(spektrumdirekt, 12.5.2003)

(55) zu dem amerikanische Filme diesen Typs niemals in der Lage sind. (DIE WELT, 7.3.2003)

(56) Wenn die Soziologie zugestehen muß, daß sie eine Gesellschaftstheorie diesen Zuschnitts bisher nicht zustandegebracht hat

(Luhmann 1997, S. 22)

Bei den einschlägigen Verbindungen von \{dies\} nimmt die Verwendung von diesen etwa 10 Prozent dieser Genitivkonstruktionen ein, bei \{jed\} nimmt diese Verwendung über das Jahrhundert hin von zehn auf zwanzig Prozent $z u$, in den IDS-Korpora überwiegen sie die mit $\{-\mathrm{es}\}$ bei weitem.

\begin{tabular}{|l|c|}
\hline jeden Jahres & 760 \\
\hline (davon:) eines jeden Jahres & 376 \\
\hline jedes Jahres & 215 \\
\hline dieses Jabres & 5.026 \\
\hline diesen Jabres & 477 \\
\hline
\end{tabular}

Die Konstruktionen mit jeden sind denen mit diesen sehr ähnlich; es gibt diese Abschnittsmarkierungen im Bezugssubstantiv (Anfang, Ende o. Ä.) und zwar durch das ganze Jahrhundert:

(57) Die Zeitschrift erscheint zu Beginn jeden Monats.

(Die Neue Gesellschaft 18.9.1907)

(58) In der ersten Woche jeden Monats werden die Wirtschafts-, Finanz-, Landwirtschafts-, Versorgungs-, Verkehrs- und Postminister zu gemeinsamen Sitzungen zusammentreten.

(Archiv der Gegenwart 18 (1948), S. 1466) 
(59) und hob am Ende jeden Satzes den Kopf, um nach seiner Frau zu schauen. (Widmer 2004, S. 65)

Analog sind auch die Qualitätsspezifizierungen in der Genitivphrase - nur in der dritten Zeitscheibe:

(60) Unter vielen Besuchern jeden Standes befinden sich Tom und Samuel (Fath 1998, S. 240)

Wenn man die Konstruktionen noch genauer betrachtet, sieht man eine zunehmende Differenzierung. Zu Beginn des Jahrhunderts finden sich neben den genannten Verwendungen Formulierungen mit den so genannten Objektsgenitiven, sowohl mit jeden wie mit jedes.

(61) durch Vereinigung mehrerer Formulare auf einer Karte, resp. allzu sparsamer Ausnutzung jeden Quadratzentimeters weißen Raumes. (Köstler 1914, S. 245-297 bzw. 258050)

(62) Jede Menge ist mit sich selbst äquivalent, wie aus der Zuordnung jedes Elementes zu sich selbst $(\mathrm{a}=\mathrm{f}(\mathrm{a}))$ hervorgeht.

(Hausdorff 1913, S. 258050)

Schon um 1950 und dann auch gegen Ende des Jahrhunderts finden sich in den Korpora Objektsgenitive nur mehr mit jedes.

(63) daß die Legitimierung jedes Herrschaftsverhältnisses stets prekär sein muß. (Dahrendorf 1957, S. 258050)

(64) Zur Vorbereitung jedes Termins kann der Vorsitzende oder ein von ihm bestimmtes Mitglied des Prozeßgerichts [...]

(Zivilprozeßordnung (ZPO) in: Schönfelder 1997, S. 23)

Dass bei \{jed\} die ,adjektivhaften Züge“ (Wiese 2009, S. 174) so stark sind, dass die schwache Flexion einigermaßen normal erscheint, zeigen auch die adjektivischen Verwendungen mit dem unbestimmten Artikel eines.

(65) „Der Verkauf war der Traum eines jeden Auktionators“, sagte Meyer im Anschluss. (Hamburger Morgenpost, 4.5.2012)

Es hängt offenbar an der Möglichkeit, die Elemente \{dies\} und mehr noch \{jed\} als adjektivartig zu verstehen, dass überhaupt das \{-en\}-Flexiv gewählt werden kann. Dabei ist die Verwendung eines jeden die gänzliche formale Klarstellung dieser Möglichkeit, die denn auch keine weiteren Einschränkungen kennt. Eine Suche in den IDS-Korpora seit 2000 ergibt weit über 10.000 Belege für diese Fügung. Wenn man die Kookkurrenzen zu sortieren versucht, kann man sehen, dass es Personenbezeichnungen sind, die den Kopf der Genitivphrase bilden. Es handelt sich zudem um Genitive in attributiven Gefügen, die mit hoher Dominanz Subjektsgenitive darstellen. An erster Stelle bei einer Kookkurrenzanalyse ist das folgende Muster genannt: 
(66) die individuelle Entscheidung [...]

\begin{tabular}{|l|l|l|l|l|}
\hline die & individuelle & Entscheidung & eines jeden & Einzelnen \\
\hline & & Entwicklung & einer jeden & \\
\hline & & Betreuung & eines jeden und einer jeden & \\
\hline & & Förderung & & \\
\hline
\end{tabular}

Aber im Prinzip ist hier die Kombinationsfähigkeit nicht eingeschränkt, und so finden sich denn unter anderem auch die bei jeden allein zu findenden Verwendungen:

(67) Zu Beginn eines jeden Schuljahres haben Eltern von schulpflichtigen Kindern erhebliche Aufwendungen für Lernmittel und sonstigen Schulbedarf. (Parlamentsprotokoll Landtag Brandenburg, 31.8.2005)

Andererseits gibt es hervorgehobene Verwendungsmuster, die von der stärkeren Akzentuierung der Bedeutung von jeder, ihrer Adjektivität lebt, es sind das zum Beispiel Genitive zu Herz, die häufig stilistisch markiert sind:

(68) [...] berührt das gefühlvolle Duett der beiden Verliebten das Herz eines jeden Zuschauers. (Mannheimer Morgen, 21.11.2003)

Als Attribut zu diesem Substantiv liegt diese Fügung zahlenmäßig deutlich vor der mit jedes, die mit jeden ist hier marginal, während das in weniger stilistisch markierten Fällen anders ist, wie die folgenden Beispiele andeuten mögen (vgl. Abb. 7):

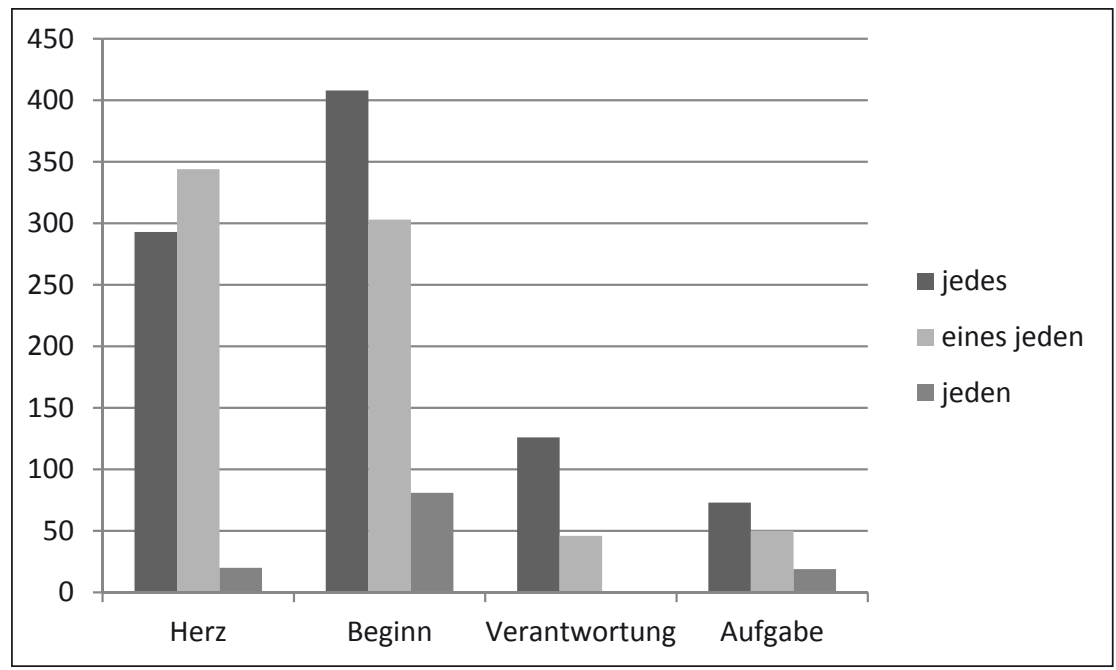

Abb. 7: Vorkommen der Konstruktionen mit \{jed-\} nach Heræ, Beginn, Verantwortung und Aufgabe 
Bei \{jed\} gibt es also eine dreifache Abstufung, bei der jedes und eines jeden vom System her gleiche Geltung haben, aber im Zweifelsfall eines jeden zur stilistischen Hervorhebung genutzt wird. In solchen, und auch den Subjektsgenitiv-Fällen, spielt die Form jeden keine bzw. kaum eine Rolle. Sie ist in ihrer Verwendung funktional beschränkt.

Auch in diesen Fällen zeigt sich, dass erstens die Fälle, in denen eine Differenzierung in der Genitivmarkierung eintritt, nicht beliebige Stellen des Systems betreffen, und dass es zweitens eine deutliche Entwicklung zu einer funktionalen Differenzierung gibt. Bei ihr haben drittens logischerweise die „abweichenden“ schwachen Formen die am stärksten eingeschränkte bzw. in lexikalische Restriktionen eingebundene Rolle.

\section{Entwicklungen im 20. Jahrhundert: Präpositionale Rektion und Präpositionalkasus}

Es ist aufgrund der formalen Verhältnisse zwischen Genitiv und Dativ nicht überraschend, dass sich an dieser Stelle verschiedene Komplikationen ergeben. Aufgrund der Reaktion in der Satzsyntax, in der Genitivobjekte keine systematische Rolle mehr spielen, und in der Nominalgruppensyntax, bei der im attributiven Bereich der morphologische Junktor Genitiv nicht in Konkurrenz zu anderen Kasus steht, kann sich das nur in der Rektion der Präpositionen niederschlagen. Und zwar betrifft das nicht alle Präpositionen, sondern die so genannten und in dieser Hinsicht recht gut beschriebenen sekundären Präpositionen. ${ }^{7}$ Generell wird festgestellt, dass sekundäre Präpositionen zur Genitiv-Verwendung neigten.

Die Frage ist, inwieweit sich hier im Verlaufe des 20. Jahrhunderts Veränderungen ergeben haben und welche Folgen das für das heutige System hat. Nicht alle Präpositionen dieses Typs sind in den Zeitscheiben unseres Korpus so häufig, dass eine statistisch einigermaßen verlässliche Analyse möglich wäre. Bei einigen haben sich auch einfach keine Veränderungen ergeben. Die folgenden Präpositionen wurden näher in Augenschein genommen.

$$
\text { anband, binnen, dank, entlang, inmitten, laut, mittels, trotz, wegen }
$$

Historisch verteilt sich das folgendermaßen auf Präpositionen mit Genitiv bzw. mit Dativ:

„Es handelt sich um sprachgeschichtlich relativ junge Bildungen, die den Weg zur typischen Präposition noch nicht vollständig zurückgelegt haben, wobei die Grammatikalisierungsstufe je nach Einzelbildung unterschiedlich ausfallen kann.“ (Di Meola 2009, S. 200); im Gefolge wird dort belegt, dass in vielen Fällen Schwankungen auftreten, die den Regelungen der Norminstanzen widersprächen. Für weitere Korpusuntersuchungen zu Rektionsschwankungen bei den Präpositionen siehe u.a. Di Meola (2000, 2002, 2005). 
(70) Genitiv: laut, mittels, statt, wegen

(71) Dativ: dank, entlang, inmitten, trotr, unweit

Wenn man nun anhand unserer Korpora für die drei Zeitscheiben zu Anfang, in der Mitte und zu Ende des Jahrhunderts überprüft, wie diese Präpositionen verwendet werden, gibt es eine Reihe von Fällen, bei denen keine Veränderung zu beobachten ist. Es handelt sich - erwartungsgemäß - im Normalfall um feste Genitivpräpositionen. Als klassischer Fall dafür mag mittels stehen, wo sich über das ganze Jahrhundert hin keine Veränderung zeigt, Ähnliches gilt für inmitten und unweit (vgl. Abb. 8).

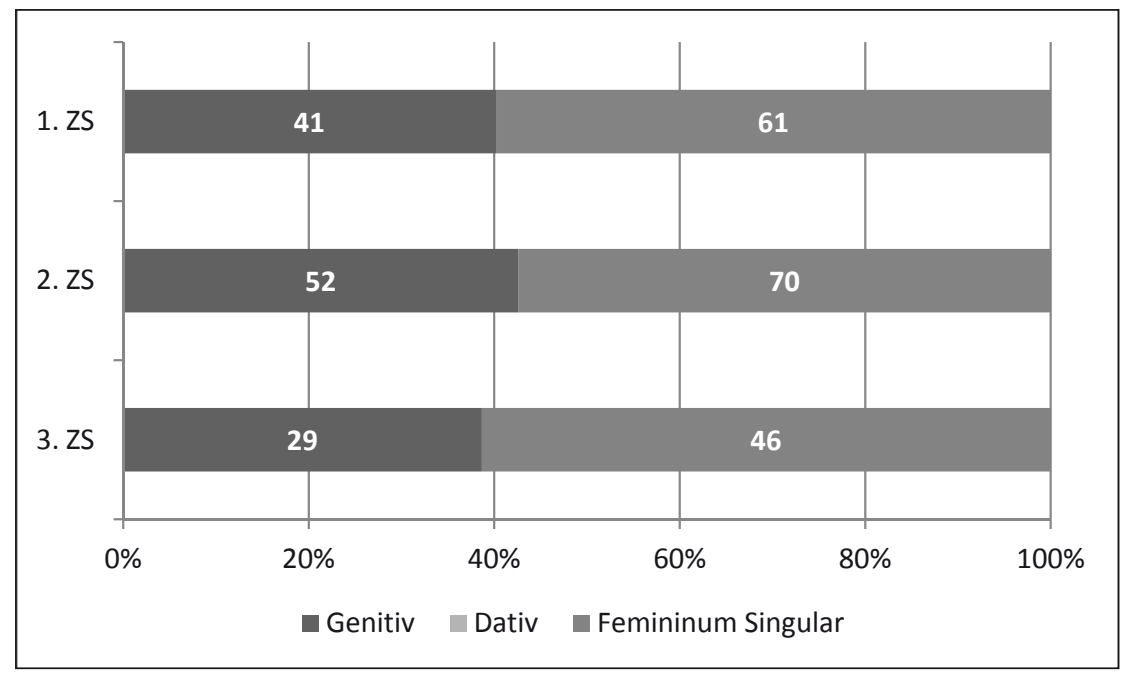

Abb. 8: Rektion der Präposition mittels (Suchanfrage: mittels + definiter/indefiniter Artikel), Vergleich Genitiv/Dativ n.s.

Zu dieser Gruppe gehört im Prinzip auch einer der Klassiker der sprachkritischen Diskussion in diesem Bereich, die Präposition wegen - gemeinsam mit ihrem semantischen Pendant trotz. (vgl. Abb. 9).

Man muss hier wie bei allen vorgeführten Belegen bedenken, dass es sich um die Verwendungen mit Artikel handelt. ${ }^{8}$ Innerhalb dieses Rahmens nun kann man feststellen, dass wir durchgehend in knapp unter 50 Prozent der Fälle Feminina im Singular vor uns haben, bei denen sich Dativ und Genitiv nicht unterscheiden, dass praktisch mehr als die Hälfte auf die klar als Genitiv erkennbaren Maskulina, Neutra und Feminina im Plural entfällt, und sich durchgehend ein kleiner Rest an Dativen findet.

8 Die Untersuchungen in Strecker (2012) zeigen auf jeden Fall, dass sich beim Einbezug der artikellosen Formen die Zahl der erkennbaren Genitive weiter vermindert. 


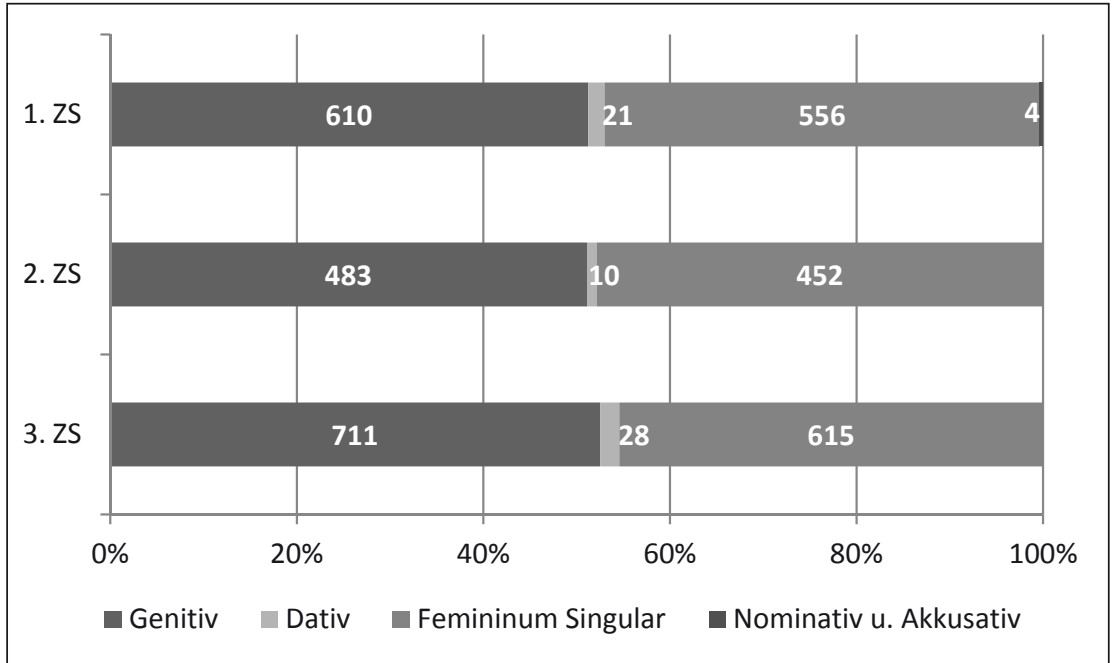

Abb. 9: Rektion der Präposition wegen (Suchanfrage: wegen + definiter/indefiniter Artikel), Vergleich Genitiv/Dativ n.s.

Wenn man die entsprechenden Dativ-Belege aus den Zeitscheiben durchsieht, so sind sie, wenn sie nicht ohnehin Wiedergabe von gesprochener Sprache sind, deutlich als stilistisch markiert gekennzeichnet:

(72) Vielleicht kommen Sie nur wegen den ekligen Bengels, aus die Sie Wurst machen wollen?" Und Unrat, errötend, nach Hilfe suchend: (Mann 1905, S. 258050)

(73) Wenn Sie wüßten, unsereiner - all die Windbeutel gäb ich mit Freuden hin für einen bessern Mann in reifern Jahren, dem es nich bloß wegen dem Amüsieren is, sondern mehr wegen dem Herzen und wegen dem Reellen [...]

(Mann 1905, S. 258050)

(74) „Wenn Gerda mit Ihnen drüber gesprochen hat, dann muß sie Ihnen doch auch gesagt haben, daß ich sie nicht heiraten kann - wegen dem Kind!“" (Horster 1950, S. 258050)

(75) „Doch nicht wegen den doofen Pömms.“ (Dölling 2003, S. 131)

(76) „Es heißt nicht, wegen den Elektrolyten“, es heißt: , wegen der Elektrolyte'.“ (Regener 2001, S. 256)

Das Gleiche gilt für die entsprechenden Belege der letzten Jahre aus den IDS-Korpora, wie etwa im folgenden Fall:

(77) Ihren Augen nicht getraut haben mehrere Autofahrer am Sonntagabend auf der Bundesstraße 9: Zwischen Schifferstadt und Limburgerhof kam ihnen ein Geisterfahrer entgegen - auf dem Fahrrad! [...] 
Manche Fahrzeuglenker mussten wegen dem falsch fahrenden Zweirad Vollbremsungen auf der stark befahrenen Bundesstraße hinlegen. (Mannheimer Morgen, 24.1.2006)

Erkennbar ist jedenfalls im Vergleich, dass sich die historische Dativ-Präposition trotz dem Genitiv-Muster völlig angepasst hat, so dass der Dativ zum Jahrhundertende keine Rolle mehr spielt (vgl. Abb. 10).?

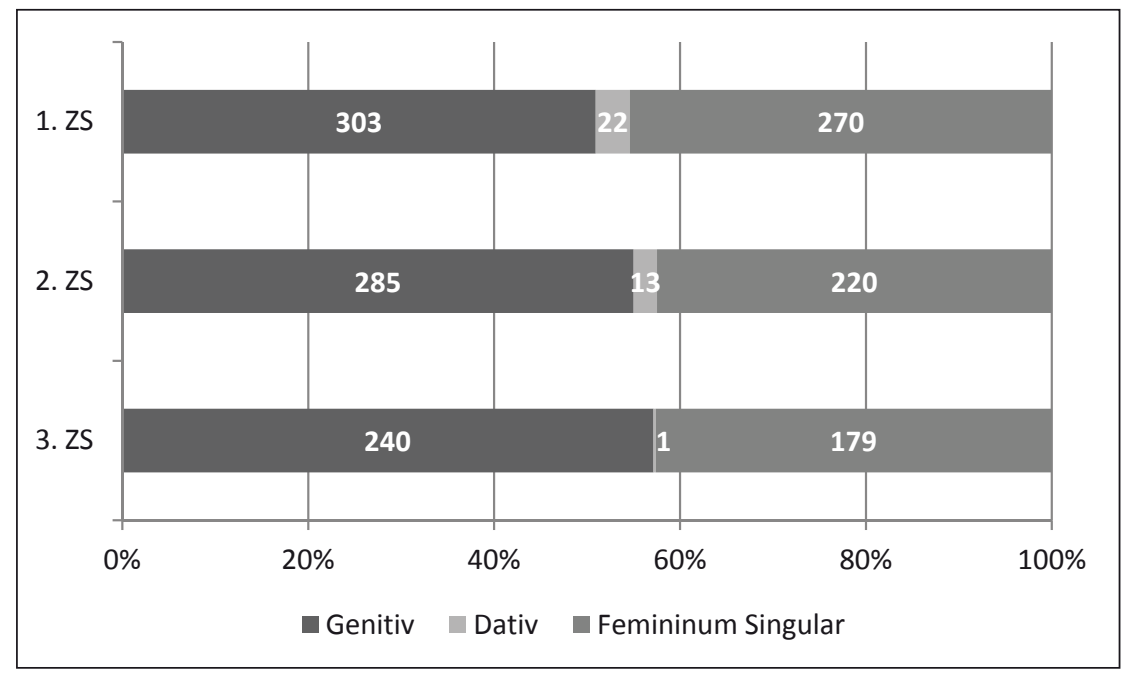

Abb. 10: Rektion der Präposition trotz, (Suchanfrage: trotz + definiter/indefiniter Artikel), Vergleich Genitiv/Dativ $\chi^{2}(2)=14,0, p<0,001$, Cramér's $V=0,13$

Den Wechsel von einer dominanten Dativ-Verwendung hin zu einer Genitiv-Verwendung (mit Dativ-Rest) hat dank mitgemacht. Diese Präposition ist daher wohl das schlagendste Beispiel dafür, wie sich im 20. Jahrhundert der Genitiv als Normalfall für diese Art von Präpositionen durchgesetzt hat - und auch hier macht die Verteilung der Genera ganz unmittelbar klar, dass diese Umschwünge dadurch erleichtert werden, dass sich bei der großen Menge der Feminina (im Singular) an der Form gar nichts ändert. In den anderen Fällen ist der Genitiv der am klarsten erkennbare Fall. Dass es um diesen Faktor gehen könnte, legt das „Ausweichen“ auf den - bei passenden Pluraltypen - ebenfalls formal erkennbaren Dativ nahe (vgl. Abb. 11).

Was trotz mit Dativ angeht, so zeigen die IDS-Korpora eine stärkere Neigung zu dieser Konstruktion in Schweizer Quellen (allerdings auch auf geringem Niveau). Auch der einzige Dativ-Beleg der dritten Zeitscheibe ist aus einem Roman des Schweizer Schriftstellers Urs Widmer: „Erfolglos, trotz einem fetten Trinkgeld“ (Widmer 1996, S. 123). 
(78) Der Stauraum in der Küche ist üppig, dank Auszügen bequem zugänglich und enthält einen Mülleimer, der Kühlschrank faßt 90 Liter. (Frankfurter Allgemeine Zeitung 25.5.1999)

(79) Die Überprüfung war von Frankreich vorgeschlagen worden, um zu verhindern, dass Kroatien trotz Mängeln bei der Bekämpfung der organisierten Kriminalität [...] Mitglied der EU werden könnte. (dpa, 23.5.2011)

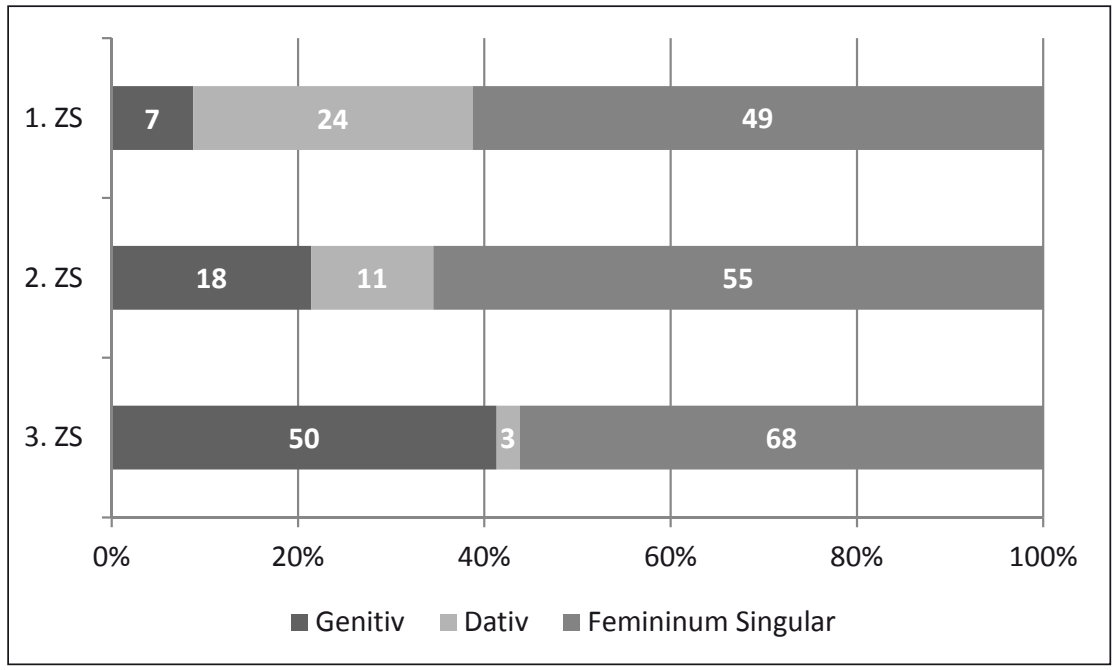

Abb. 11: Rektion der Präposition dank (Suchanfrage: dank + definiter/indefiniter Artikel), Vergleich Genitiv/Dativ $\chi^{2}(2)=45,4, p<0,001$, Cramér's $V=0,63$

Diese Entwicklungen scheinen in die Richtung zu laufen, die unterdifferenzierte Femininform im verbalen Kontext normalerweise als Dativ zu lesen, und im attributiven und präpositionalen Kontext als Abhängigkeitsmarker - außer wo eine funktionale Dativ-Interpretation greift bzw. die historische Motivation noch stark ist.

Solch einen Fall scheint die Präposition laut darzustellen, die sich als vergleichsweise amtssprachliche Präposition in ihrer Rektion auf Präpositionen stützt, die eine ähnliche Funktion haben. So weist das Paul'sche Wörterbuch (Paul 2002, S. 595) unter dem Stichwort laut darauf hin, dass noch im 19. Jahrhundert die Fügung nach Laut des Briefes üblich war, aus der seit dem 15. Jahrhundert „laut als Präp. mit Gen. [hervorgegangen sei,] wofür zuweilen (durch Einfluß von nach, vgl. Behaghel, Synt. 2,31.48) auch der Dat. erscheint" - was immerhin mit einem Goethe-Zitat belegt wird.

Somit zeigt sich in diesem letzten beobachteten Fall die Tendenz, bei dieser Gruppe von Präpositionen den Genitiv, und damit auch seine gut erkennbaren Formen des starken Maskulinum und Neutrum als Signal der 
Regiertheit zu nutzen, gemeinsam mit der entsprechenden Nutzung des formal erkennbaren Dativs des Plural könnte man von einem Nutzen der jeweils erkennbarsten Abhängigkeitsform sprechen - und damit auch in diesen Fällen von einer funktionalen Nutzung einer formalen Unterscheidung in den Fällen, in denen sie nicht (mehr) semantisch lesbar ist.

\section{Schluss}

Wir haben drei Fälle von grammatischen Veränderungen über das 20. Jahrhundert hin betrachtet, an denen gezeigt werden konnte, dass Entwicklungen und Verwendungsweisen, die generelle Tendenzen des Wandels des Deutschen betreffen, nicht einfach zum Verschwinden von Dingen oder zu beliebigen „Abweichungen“ von einer angenommenen Norm führen. Vielmehr kommt es in allen Fällen zu einer funktionalen Ausdifferenzierung zwischen der Verwendung „alter“ und „,neuer" Konstruktionen. Die generellen Tendenzen des Wandels führen zu Formen, in denen die morphologische Kennzeichnung der Kasus an den flektierten „Begleitern“ des Substantivs - Determinativen bzw. Adjektiven - auftritt, und nicht am Substantiv. Die Durchsetzung des Prinzips der Monoflexion betrifft auch die interne morphologische Steuerung der Morphologie zwischen diesen nicht-substantivischen Elementen der Nominalgruppe. Das führt etwa zu einer sehr differenzierten und kontextfunktionalen Differenzierung bei Übergangsfällen (z.B. jedes/jeden Jahres). Die überdifferenzierten Kongruenz-Konstruktionen, die sich im Kontext dieser flexivischen Verschiebungen ergeben (\{-es\}Genitive; $\{$-e $\}$-Dative) werden aber nicht einfach aufgegeben, sondern als Signal für spezifische Fälle der Verwendung genutzt, in denen ihre deutlichen formalen Signale als Markierungen nützlich sind, das hat bei den insgesamt marginaleren Dativ-Formen eher stilistischen, bei den in dieser Hinsicht stabilen Genitiv-Formen aber eine systematische Umdeutung als starkes Abhängigkeits- bzw. Rektionssignal im nicht-verbalen Bereich zur Folge, einerseits im Kontext der nominalen Attribute, andererseits im Bereich einer bestimmten Klasse von Präpositionen.

Um die Entwicklungen im Flexionssystem wie die hier angesprochenen angemessen beurteilen zu können, sind drei Betrachtungsweisen vonnöten, die der alltäglichen Beobachtung einer Veränderung einen Rahmen geben, der solche Erscheinungen erst zu beurteilen erlaubt. Zum einen braucht es einen makroskopischen Blick auf die Stellung der einzelnen Veränderungen in oder in Bezug auf langfristige Entwicklungstendenzen, die ja eher der Systemstabilisierung dienen. Zum anderen bedarf es aber unmittelbar ergänzend eines mikroskopischen Blicks, der möglichst genau die Bedingungen einer schwankenden Verwendung - von Variation - zu kontextualisieren 
erlaubt. Und zum dritten bedarf es - und das 20. Jahrhundert mag in Länge und Stellung in der Entwicklung der deutschen Standardsprache einen angemessenen Zeitausschnitt darstellen - dieser Beobachtung im Hinblick auf eine Zeitstrecke, die Variation und Wandel in ihren Richtungen zu beurteilen erlaubt.

Zumindest im Hinblick auf die vorgeführten Ausschnitte ergibt sich als Ergebnis dieses dreifachen Blicks, dass nicht einfach Elemente verschwinden, die man eigentlich noch bräuchte, sondern dass über eine Zeit laufende Umstellungen in dem, was als zentrales und marginales Kodierungsmodell gelten kann, dazu führt, dass der Gewinn an Merkmalhaftigkeit, der mit Marginalität korreliert, als Markiertheitssignal genutzt wird.

\section{Literatur}

Braun, Heinrich (1775): Heinrich Brauns Anleitung zur deutschen Sprachkunst zum bequemern Gebrauche von ihm selbst ins Kurze gezogen. 3. u. verb. Aufl. München.

Deutsche Akademie für Sprache und Dichtung/Union der deutschen Akademien der Wissenschaften (Hg.) (2013): Reichtum und Armut der deutschen Sprache. Erster Bericht zur Lage der deutschen Sprache. Berlin/Boston.

Di Meola, Claudio (2000): Die Grammatikalisierung deutscher Präpositionen. (= Studien zur deutschen Grammatik 62). Tübingen.

Di Meola, Claudio (2002): Präpositionale Rektionsalternation unter dem Gesichtspunkt der Grammatikalisierung: das Prinzip der „maximalen Differenzierung“. In: Cuyckens, Hubert/Radden, Günter (Hg.): Perspectives on prepositions. (= Linguistische Arbeiten 454). Tübingen, S. 101-129.

Di Meola, Claudio (2005): Entwicklungstendenzen im deutschen Präpositionalsystem: Rektion ,entsprechend der Regeln' oder, wider den Normen'? In: Di Meola, Claudio/Hornung, Antonie/Rega, Lorenza (Hg.): Perspektiven Eins. Akten der 1. Tagung Deutsche Sprachwissenschaft in Italien (Rom, 6.-7.2.2004). Rom, S. 251-267.

Di Meola, Claudio (2009): Rektionsschwankungen bei Präpositionen - erlaubt, verboten, unbeachtet. In: Konopka/Strecker (Hg.), S. 195-221.

Eichinger, Ludwig M. (2011): Normprobleme, oder: Variation ist sinnvoll. Überlegungen zum heutigen Deutsch. Stuttgart.

Eichinger, Ludwig M. (2012): Gutes Weines, frohes Muthes, reines Herzens - Geschichten vom Genitiv. In: Konopka/Schneider (Hg.), S. 83-104.

Eichinger, Ludwig M. (2013): Die Entwicklung der Flexion: Gebrauchsverschiebungen, systematischer Wandel und die Stabilität der Grammatik. In: Deutsche Akademie für Sprache und Dichtung/Union der deutschen Akademien der Wissenschaften (Hg.), S. 121-170.

Heringer, Hans Jürgen (2012): Anfang diesen Jahres? - Anders gefragt. In: Konopka/ Schneider (Hg.), S. 65-70. 
Konopka, Marek (2012): Dem Manne kann geholfen werden - Wann kommt das Dativ-e zum Einsatz? In: Konopka/Schneider (Hg.), S. 115-123.

Konopka, Marek/Schneider, Roman (Hg.) (2012): Grammatische Stolpersteine digital. Festschrift für Bruno Strecker zum 65. Geburtstag. Mannheim.

Konopka, Marek/Strecker, Bruno (Hg.) (2009): Deutsche Grammatik - Regeln, Normen, Sprachgebrauch. (= Jahrbuch des Instituts für Deutsche Sprache 2008). Berlin/New York.

Klügel, Georg Simon (1809): Encyklopädie oder zusammenhängender Vortrag der gemeinnützigsten Kenntnisse. Teil 5: Die deutsche Sprachlehre. 3., unverb. u. verm. Ausg. Berlin/Stettin.

Lindqvist, Christer (1994): Zur Entstehung von Präpositionen im Deutschen und Schwedischen. (= Linguistische Arbeiten 311). Tübingen.

Paul, Hermann (2002): Deutsches Wörterbuch. Bedeutungsgeschichte und Aufbau unseres Wortschatzes. 10, überarb. u. erw. Aufl. Tübingen.

Sahel, Said (2011): Monoflexion als Erklärung für Variation in der Nominalphrasenflexion. In: Konopka, Marek et al. (Hg.): Grammatik und Korpora 2009. Dritte Internationale Konferenz (Mannheim 22.-24.09.2009). (= Korpuslinguistik und interdisziplinäre Perspektiven auf Sprache 1). Tübingen, S. 485-494.

Stenschke, Oliver (2007): „Ende diesen Jahres“: Die Flexionsvarianten von Demonstrativpronomina als ein Beispiel für Degrammatikalisierung. In: Deutsche Sprache 35, S. $63-85$.

Strecker, Bruno (2006): Anfang diesen Jahres oder Ende dieses Jahres? - Genitiv Singular beim Demonstrativ-Artikel. In: Sprachreport 22, 3, S. 13-15.

Strecker, Bruno (2012): Wegen dem Regen oder wegen des Regens - Dativ oder Genitiv? Internet: http://hypermedia.ids-mannheim.de/call/public/fragen.ansicht?v_id=67 (Stand: 4.11.2013).

Takada, Hiroyuki (1998): Grammatik und Sprachwirklichkeit von 1640-1700. Zur Rolle deutscher Grammatiker im schriftsprachlichen Ausgleichsprozeß. (= Reihe Germanistische Linguistik 203). Tübingen.

Wiese, Bernd (2009): Variation in der Flexionsmorphologie: Starke und schwache Adjektivflexion nach Pronominaladjektiven. In: Konopka/Strecker (Hg.), S. 166-194. 
